\title{
$B(H)$ IS VERY NONCOMMUTATIVE
}

PAUL WILLIG

For a $W-^{*}$ algebra the following two properties are partial forms of commutativity.

Definition 1. A $W-^{*}$ algebra $a$ has property $L$ if there are unitary operators $U_{n} \in Q$ such that $U_{n} \rightarrow 0$ weakly and such that for every $A \in Q, U_{n} A U_{n}^{*} \rightarrow A$ strongly.

Definition 2. A $W-^{*}$ algebra $a$ has property $A . A$. if there are *-automorphisms $\phi_{n}$ of $a$ such that for every pair $S, T \in Q,\left[S, \phi_{n}(T)\right]$ $\rightarrow 0$ strongly, where $[S, T]=S T-T S$.

Definition 1 is due to Pukánszky [3] and Definition 2 is essentially due to Sakai [4].

Let $H$ denote a separable Hilbert space and let $B(H)$ denote the algebra of all bounded operators on $H$. It is our aim to prove that $B(H)$ has neither property A.A. nor property $\mathrm{L}$.

The author wishes to thank H. M. Hilden for suggesting Lemma 3, and expresses particular thanks to S. Sakai for making available a preprint of [4] and for improving the proof of Lemma 3.

Lemma 3. Let $V_{n}$ be a sequence of unitary operators such that $V_{n}^{*} A V_{n}$ $\rightarrow A$ strongly for each $A \in B(H)$. Then there is a subsequence (again called $V_{n}$ ) and a scalar $\lambda$ such that $|\lambda|=1$ and $V_{n} \rightarrow \lambda I$ strongly.

Proof. Clearly $A V_{n}-V_{n} A \rightarrow 0$ strongly. By the weak compactness of the unit ball of operators we may assume that $V_{n}$ (or a subsequence) converges weakly to some $V$. It follows readily that $A V=V A$ for all $A \in B(H)$ and hence that $V=\lambda I,|\lambda| \leqq 1$.

Let $\left\{x_{n}\right\}$ be an orthonormal basis for $H$ and define an operator $A$ such that $A x_{1}=x_{1}, A x_{j}=0, j>1$. A simple calculation shows that

$$
\left(\left(V_{n}^{*} A V_{n}-A\right) x_{1}, x_{1}\right)=\left|\left(V_{n} x_{1}, x_{1}\right)\right|^{2}-1 \rightarrow 0 .
$$

Since clearly $\left(V_{n} x_{1}, x_{1}\right) \rightarrow \lambda$, we see that $|\lambda|^{2}=1$. Thus $|\lambda|=1$. Since the $V_{n}$ are unitary and converge weakly to the unitary $\lambda I$, it follows that $V_{n} \rightarrow \lambda I$ strongly. Q.E.D.

Corollary 4. $B(H)$ does not have property $\mathrm{L}$.

Proof. Use Lemma 3 putting $V_{n}=U_{n}^{*}$. Q.E.D.

Theorem 5. $B(H)$ does not have property A.A.

Received by the editors May 16, 1969. 
Proof. Since it is well known that every *-automorphism of $B(H)$ is inner (see [1]) we may assume that $\phi_{n}(A)=U_{n} A U_{n}^{*}$ for some unitary $U_{n}$. Suppose therefore that $B(H)$ has property A.A. and that $\left\{U_{n}\right\}$ is the corresponding sequence of unitaries demonstrating the property. It follows that for each pair $A, B \in B(H)$ we have $U_{n} A U_{n}^{*} B$ $-B U_{n} A U_{n}^{*} \rightarrow 0$ strongly.

Let $E$ denote the orthogonal projection on the span of $\left\{x_{1}, x_{3}, \cdots\right\}$, and let $F=I-E$. Then there is a unitary $V$ such that $E=V F V^{*}$, and there are operators $P, Q, R$, and $S$ such that $I=S E R=P F Q$. Let $\left\{V_{m}\right\}$ be a countable set of unitaries such that $E, F, P, Q, R, S$, and $V$ are linear combinations of the $V_{m}$ and such that $\left\{V_{m}\right\}$ is closed under multiplication and taking adjoints. Let $Q$ be the involutive algebra generated by the $\left\{V_{m}\right\}$. We may assume that the $U_{n}$ are such that the strong limit of the sequence $U_{n} V_{m} U_{n}^{*}=\lambda_{m} I$ exists for each $V_{m}$, by Lemma 3 and the Cantor diagonal process. Then it is readily verified that if $A \in Q, A=\sum_{m=1}^{N} a_{m} V_{m}$, we may define $\phi: Q \rightarrow C$ by $\phi(A)=\lambda$ where strong limit $U_{n} A U_{n}^{*}=\lambda I$. It is easy to check that $\phi$ is linear, multiplicative, and $\phi(I)=1$. Hence $\phi(E)=0$ or $\phi(E)=1$. But $\phi(F)$ $=\phi(V) \phi(E) \phi\left(V^{*}\right)$, and therefore $\phi(F)=\phi(E)$. Therefore $\phi(I)$ $=\phi(E)+\phi(F)$, so that $\phi(I)=0$ or $\phi(I)=2$, both contradictions. It follows that $B(H)$ does not have property A.A. Q.E.D.

This proof is essentially the proof of Theorem 4.1 in [2].

\section{REFERENCES}

1. J. Dixmier, Les algèbres d'opérateurs dans l'espace Filbertien, Cahiers scientifiques, Fasc. XXV. Gauthier-Villars, Paris, 1957. MR $20 \# 1234$.

2. H. Porta and J. T. Schwartz, Representations of the algebra of all operators in Hilbert space, and related analytic function algebras, Comm. Pure Appl. Math. 20 (1967), 457-492. MR 35 \#2157.

3. L. Pukánszky, Some examples of factors, Publ. Math. Debrecen 4 (1956), 135156. MR 18, 323.

4. S. Sakai, Asymptotically Abelian $I I_{1}$-factors (to appear).

Stevens Institute of Technology 